\title{
Non-CF bronchiectasis: does knowing the aetiology lead to changes in management?
}

\author{
A.M. Li**\#, S. Sonnappa ", C. Lex*, E. Wong\#, A. Zacharasiewicz*, \\ A. Bush* and A. Jaffe
}

ABSTRACT: The aim of the current study was to review the aetiology of non-cystic fibrosis (CF) bronchiectasis from two tertiary paediatric respiratory units in order to determine how often making a specific aetiological diagnosis leads to a change in management, and to assess the contribution of computed tomography (CT) in determining the underlying diagnosis.

The case records of all patients who were diagnosed as having bronchiectasis by CT, currently being seen at the Royal Brompton Hospital and Great Ormond Street Hospital for Children (London, UK), were reviewed. All patients had undergone extensive investigations, and the underlying aetiology and the area of pulmonary involvement (as seen on CT) were recorded. A total of 136 patients were identified; there were 65 young males and the group median (range) age was 12.1 yrs (3.1-18.1). Immunodeficiency, aspiration and primary ciliary dyskinesia accounted for $67 \%$ of the cases. In 77 (56\%) children, the identification of a cause led to a specific change in management. There was no association between aetiology and the distribution of CT abnormalities.

In conclusion, immunodeficiency and other intrinsic abnormalities account for the majority of cases of non-cystic fibrosis bronchiectasis seen in the current authors' units. Computed tomography scans do not contribute towards identifying the aetiology and, most importantly, a specific aetiological diagnosis frequently leads to a change in management.

KEYWORDS: Bronchiectasis, computed tomography, immunodeficiency, primary ciliary dyskinesia

B ronchiectasis was first described as "production of fetid sputum along with bronchial dilatation" [1]. It is a pathological description of a disease process that has many possible causes. The characteristic features are abnormally dilated thick-walled bronchi that are inflamed and chronically infected by bacteria. It is a chronic condition, which can result in significant physical and social morbidity.

In previous large series, the majority of cases were due to extrinsic factors, especially childhood respiratory infections (severe pneumonia, pertussis, complicated measles and tuberculosis (TB)) as the cause of permanent bronchial damage. SingLETON et al. [2] reviewed the case histories of 46 Alaskan native children with bronchiectasis born in the 1970s, and concluded that recurrent pneumonia was the major preceding medical condition leading to bronchial damage. In a study by EASTHAM et al. [3], previous pneumonia was the most common cause found in 93 cases of non-cystic fibrosis (CF) bronchiectasis. Likewise, nearly $50 \%$ of children were found to have developed bronchiectasis after TB or severe pneumonia in a review study by KARAKOC et al. [4]. Nowadays, with early immunisation and the widespread use of antibiotics in childhood, post-infectious damage is likely to be less relevant. The emphasis has now changed to the investigation of intrinsic defects or noninfective extrinsic insults that predispose to bronchial inflammation or infection. These include congenital defects, aspiration of irritants, immunodeficiency and mucociliary clearance problems. The standard medical treatment of underlying bronchiectasis usually involves airway clearance techniques, antibiotics, antiinflammatory medication and bronchodilators (where appropriate). Detailed investigations are often carried out to determine the underlying cause of the condition, but there is little point

\section{AFFILIATIONS}

*Dept of Paediatric Respiratory Medicine, Royal Brompton Hospital, and

'Dept of Paediatric Respiratory Medicine and Portex Unit, Great Ormond Street Hospital and Institute of Child Health, London, UK.

\#Dept of Paediatrics, Prince of Wales Hospital, Chinese University of Hong Kong, Shatin, Hong Kong.

CORRESPONDENCE

A. Jaffe

Portex Respiratory Medicine Unit

Level 6

Cardiac Wing

Great Ormond Street Hospital for Children and Institute of Child Health Great Ormond Street

London WC1N 3JH UK

Fax: 442078298634

E-mail: A.Jaffe@ich.ucl.ac.uk

Received:

November 082004

Accepted after revision:

March 292005 
unless they result in a change in management. The expense and inconvenience of an investigation may be considerable, but the value of detailed investigations into the cause of bronchiectasis has, however, not been evaluated.

High-resolution computed tomography (HRCT) has proven to be a reliable and noninvasive method for the assessment of bronchiectasis, and has largely eliminated the need for bronchography [5]. HRCT can accurately diagnose bronchiectasis, localise and describe areas of parenchymal abnormality, and identify bronchiolar abnormalities and mucus plugging to the level of fifth- and sixth-order bronchi. It can also identify focal areas of air trapping as an indication of small airway disease $[5,6]$. It is commonly considered as the technique of choice in the diagnosis of bronchiectasis, but controversy still exists as to whether the distribution of bronchiectasis seen in different diseases is characteristic enough to suggest a specific diagnosis [7-10].

The aim of this study is three-fold: 1) to review the aetiology of cases with non-CF bronchiectasis from two tertiary paediatric respiratory units; 2) to determine how often making a specific diagnosis leads to a change in management; and 3) to assess whether the specific causes of bronchiectasis could be differentiated on the basis of the localisation of abnormalities on HRCT.

\section{METHODS}

\section{Study subjects}

The study population consisted of outpatients currently attending the paediatric respiratory clinics at both the Royal Brompton Hospital and Great Ormond Street Hospital for Children (London, UK). In this study, children aged $\leqslant 18$ yrs were retrospectively identified by a computerised database, using the following key diagnoses: bronchiectasis, chronic suppurative lung disease, and chronic cough. Patients who presented during the period of 1986-2002 were identified. The identified cases were checked by the two of the present authors (A.M. Li and S. Sonnappa) to ascertain whether they satisfied the following inclusion criteria: HRCT-diagnosed bronchiectasis in subjects who presented with suggestive clinical features, and the diagnosis of CF excluded by sweat test and/or analysis of genetic mutations seen in the local population [11]. If any doubt existed, nasal potential differences [12] and faecal elastase [13] were performed to determine that CF was not the cause of the bronchiectasis.

A radiological diagnosis of bronchiectasis was made on the HRCT if any of the following features were present [5]: 1) a lack of normal bronchial tapering in cuts parallel to the direction of travel on sequential slices; 2) bronchi having an internal diameter greater than the diameter of the accompanying pulmonary artery; and 3) dilated bronchi visible adjacent to the nonmediastinal pleura.

\section{Study design}

The case records of children satisfying the inclusion criteria for recruitment were retrospectively reviewed, using a standardised clinical data extraction form. The following data were recorded: current age, initial presenting symptoms, and the specific diagnosis of cause of the bronchiectasis. From the HRCT scan performed at diagnosis, the presence or absence of bronchiectasis was recorded in each lobe, with the lingula being considered as a separate lobe. Widespread disease was defined as bronchiectactic involvement of five or more lobes. All radiological assessments were performed without knowledge of the findings of the aetiological work-up.

Each child was investigated to determine the underlying aetiology at the discretion of the paediatric respirologist in charge, and not all children underwent all these tests. The investigations included: 1) measurement of serum immunoglobulins (Ig), IgG subclasses, complement levels, specific antibody responses to tetanus toxoid, capsular polysaccharides of Haemophilus influenzae type b and Streptococcus pneumoniae, lymphocyte subsets, antigen/mitogen stimulation tests, staphylococcal and candida killing-ability tests and HIV screening test. Subsequently, the opinion of an immunologist was sought if further specific immunological investigations were required; 2) serum $\alpha_{1}$-antitrypsin level and genotype if abnormal; 3) bacterial culture of cough swab and sputum and their results noted; 4) Mantoux test; 5) 24-h oesophageal $\mathrm{pH}$ monitoring; 6) bronchoscopy and bronchoalveolar lavage, with bacterial culture and cytology for lipid-laden macrophages; and 7) nasal ciliary beat frequency using light microscopy [14]. In the absence of coordinated ciliary beating of normal frequency and pattern, or if the history was particularly suggestive, a brushing was sent for electron microscopy. Doubtful results led to repeat brushing after treating any local infection. In cooperative subjects, nasal nitric oxide was also measured [15, 16]. Genetic studies were, however, not routinely available; and the following techniques were used in certain situations: 8) if aspiration or gastrointestinal anomalies were suspected, a barium meal would be given; 9) if dysfunctional swallowing was suspected, videofluoroscopy was performed; and 10) in cases who were cooperative, spirometry would be performed according to the American Thoracic Society guidelines [17]. Three technically acceptable manoeuvres were performed each time, and the highest value of forced expiratory volume in one second (FEV1) and its corresponding forced vital capacity (FVC) were recorded. Values were expressed as a percentage of predicted normal values. Bronchiectasis was defined as idiopathic if extensive investigations failed to reveal an underlying aetiology. Approval was obtained from local ethics committee of the two hospitals to carry out this retrospective review.

\section{Statistical analysis}

The demographic data and lung function parameters were expressed as median and median percentage predicted, respectively.

\section{RESULTS}

A total of 136 patients were identified. There were 65 males, and the group median age was 12.1 yrs (range 3.1-18.1) at the time of the study. One hundred cases were referred for investigations by their local hospital paediatricians. An aetiology for the bronchiectasis was identified in 101 patients, whereas no cause could be established in 35 . The identified causes of bronchiectasis are shown in table 1 . For the group as a whole, at diagnosis, the median (range) FEV1 was $71 \%$ (15$133)$ and FVC was $77 \%$ (14-122). The diagnostic yield of each investigation is shown in table 2 . In 77 patients, the cause 


\begin{tabular}{|c|c|c|c|}
\hline Aetiology & $\begin{array}{c}\text { Subjects } \\
n\end{array}$ & $\mathrm{FEV}_{1}$ & FVC \\
\hline Immunodeficiency & 46 & $75(15-133)$ & $76(14-122)$ \\
\hline \multicolumn{4}{|l|}{ Primary immune defects } \\
\hline Panhypogammaglobulinaemia & 3 & & \\
\hline Hyper-lgE syndrome & 2 & & \\
\hline Mannose-binding protein deficiency & 1 & & \\
\hline Qualitative antibody deficiency & 1 & & \\
\hline $\lg G$ deficiency & 4 & & \\
\hline Combined variable immunodeficiency & 14 & & \\
\hline Undefined combined immunodeficiency & 6 & & \\
\hline X-linked agammaglobulinaemia & 4 & & \\
\hline MHC class-2 deficiency & 1 & & \\
\hline B-cell deficiency & 1 & & \\
\hline Chronic mucocutaneous candidiasis & 1 & & \\
\hline Chronic granulomatous disease & 1 & & \\
\hline Wiskott-Aldrich syndrome & 1 & & \\
\hline \multicolumn{4}{|l|}{ Secondary immune defects } \\
\hline Post-chemotherapy & 1 & & \\
\hline AIDS & 5 & & \\
\hline Idiopathic & 35 & $69(34-104)$ & $78(51-116)$ \\
\hline Aspiration & 25 & $71(33-91)$ & $69(36-105)$ \\
\hline Primary ciliary dyskinesia & 20 & $66(39-100)$ & $82(46-111)$ \\
\hline Childhood respiratory infection & 5 & $39(26-62)$ & $64(42-76)$ \\
\hline Measles & 2 & & \\
\hline Pertussis & 1 & & \\
\hline Adenovirus & 1 & & \\
\hline Tuberculosis & 1 & & \\
\hline Congenital structural malformation & 5 & $83(78-85)$ & $92(84-98)$ \\
\hline Congenital lobar emphysema & 1 & & \\
\hline Pulmonary artery sling & 1 & & \\
\hline Bronchial atresia with bronchocoele & 1 & & \\
\hline Yellow nail syndrome & 1 & & \\
\hline Bronchomalacia & 1 & & \\
\hline
\end{tabular}

Data are presented as median percentage predicted (range), unless otherwise stated. FEV1: forced expiratory volume in one second; FVC: forced vital capacity; Ig: immunoglobulin; MHC: major histocompatibility complex.

identified had implications for prognosis and treatment. The patients with combined variable immunodeficiency and others with hypogammaglobulinaemia or agammaglobulinaemia were given Ig replacement therapy. Patients identified to have aspiration were treated with intensive acid-suppression and prokinetic agents; some went on to have Nissen's fundoplication with/without gastrostomy. Patients identified to have primary ciliary dyskinesia (PCD) were seen by a specialist ear, nose and throat (ENT) surgeon in a joint PCD clinic, allowing appropriate management to be instigated [18].

The predominant symptoms at the time of diagnosis are listed in table 3. The most common reason for referral was recurrent "chest infection", and this was the presenting symptom in over half of the cases.

The distribution of bronchiectasis in relation to the specific aetiology is shown in table 4 . In patients with immunodeficiency,
TABLE 2 Diagnostic yield of each individual investigation

\begin{tabular}{|c|c|c|}
\hline Investigation & Performed $\mathbf{n}$ & Positive $n$ \\
\hline Immunology work-up & 110/136 (81) & $46 / 110(42)$ \\
\hline $\begin{array}{l}\text { Bronchoscopy with } \\
\text { alveolar lavage }\end{array}$ & $68 / 136(50)$ & $\begin{array}{l}\text { 8/68 (12); two cases with } \\
\text { laryngomalacia, one case } \\
\text { with tracheobronchial malacia, } \\
\text { one case with bronchial atresia } \\
\text { showed distorted anatomy, one } \\
\text { case with aspiration showed } \\
\text { inflamed right middle bronchus, } \\
\text { and three cases with aspiration } \\
\text { had a significant amount of } \\
\text { fat-laden macrophages detected }\end{array}$ \\
\hline Ciliary function & $66 / 136(49)$ & 19/66 (29) \\
\hline Nasal nitric oxide & 19/136 (14) & 9/19 (47); range $16.3-133 \mathrm{ppb}$ \\
\hline Ciliary beat frequency $\#$ & $17 / 20(85)$ & $\begin{array}{l}\text { 11/17 showed static ciliary } \\
\text { motion; } 4 / 17 \text { showed } \\
\text { reduced beat frequency; } \\
\text { 2/17 showed dyskinetic } \\
\text { ciliary motion }\end{array}$ \\
\hline Electron microscopy ${ }^{\#}$ & $13 / 20(70)$ & $\begin{array}{l}\text { 2/13 showed orientation defect; } \\
\text { 5/13 showed IDA abnormalities; } \\
\text { 2/13 showed ODA abnormalities; } \\
\text { 4/13 showed combined IDA and } \\
\text { ODA abnormalities }\end{array}$ \\
\hline 24-h pH study & $51 / 136(38)$ & $23 / 51(45)$ \\
\hline$\alpha_{1}$-Antitrypsin & 30/136 (22) & $0 / 27(0)$ \\
\hline Mantoux test & 23/136 (17) & 0/23 (0) \\
\hline Barium contrast study & 18/136 (13) & $\begin{array}{c}\text { 2/18 (11); both showed } \\
\text { presence of reflux, but, in } \\
\text { one case, } \mathrm{pH} \text { study was normal }\end{array}$ \\
\hline
\end{tabular}

Data are presented as $\mathrm{n} / \mathrm{N}(\%)$. ppb: parts per billion; IDA: inner dynein arm; ODA: outer dynein arm. ${ }^{\#}$ : for the 20 primary ciliary dyskinesia patients.

nine out of $46(20 \%)$ showed widespread disease, and the commonest sites were the middle $(80 \%)$ and lower lobes $(91 \%)$. Bilateral disease was seen in 38 out of $47(83 \%)$ cases. In idiopathic cases, widespread bronchiectasis occurred in $9 \%$,

\section{TABLE 3 Symptom history at the time of diagnosis of bronchiectasis}

Symptom history

Cases n

Recurrent chest infections

105

Chronic cough

Recurrent wheeze

47

Gastro-oesophageal reflux

Rhinitis from neonatal period

Recurrent otitis media

Failure to thrive

Exercise intolerance

14

11

7

7

6

2

$n=136$ 
TABLE 4 Computed tomography distribution of bronchiectasis

\begin{tabular}{|c|c|c|c|c|c|c|c|}
\hline Immunodeficiency & 20 & 37 & 42 & 9 & 8 & 38 & 28 \\
\hline Aspiration & 9 & 13 & 20 & 6 & 10 & 15 & 14 \\
\hline PCD & 5 & 17 & 18 & 0 & 5 & 15 & 15 \\
\hline Congenital malformations & 3 & 2 & 3 & 0 & 4 & 1 & 1 \\
\hline
\end{tabular}

Data are presented as $\mathrm{n}$. UL: upper lobe involvement; ML: middle lobe involvement; LL: lower lobe involvement; widespread: involvement of $\geqslant 5$ lobes; unilateral: involvement of only one side; bilateral: involvement of both sides; bilateral symmetrical: involvement of identical lobes on both sides; PCD: primary ciliary dyskinesia.

with a predominant middle $(71 \%)$ and lower $(80 \%)$ lobe involvement, similar to the immunodeficiency pattern. No PCD patient had widespread disease and, again, there was middle and lower lobe predominance. In cases with bronchiectasis secondary to aspiration, the disease was mainly $(80 \%)$ seen in the lower lobes. In cases with congenital structural malformations and bronchiectasis secondary to childhood infection, there was no predominant lobar involvement; however, the numbers in these groups were small. Single lobe involvement was more commonly seen in subjects with aspiration and idiopathic bronchiectasis, but the distribution was, once again, not specific to a particular aetiology. There was a great deal of overlap between the underlying aetiology of bronchiectasis and computed tomography (CT) distribution of the abnormalities, and no association between the modalities could be established.

The microbiological flora associated with each diagnosis are shown in table 5. The commonest organisms isolated were: $H$. influenzae, S. pneumoniae, Pseudomonas aeruginosa, Staphylococcus aureus, and methicillin-resistant $S$. aureus. Two or more organisms were isolated in only a small proportion of patients, and the commonest pair of organisms isolated was $H$. influenzae and S. pneumoniae. Fungi (Candida albicans) were only isolated from patients with immunodeficiency. There were no significant differences in spirometry between patients with the same diagnosis but different microbial infection, or between patients with the same microbial infection but different diagnoses.

\section{DISCUSSION}

A total of 136 patients with bronchiectasis confirmed on HRCT were reviewed, and their aetiological factors were characterised. A specific cause was identified in 101 patients, but, more importantly, in 77 patients, the cause identified led to a distinct and individualised change in management. The current authors have also demonstrated that the distribution of HRCT abnormalities had no correlation with the underlying cause of bronchiectasis. It should be noted that there was quite marked airflow obstruction already present at the time of diagnosis in some of the patients. Therefore, the exclusion of $\mathrm{CF}$ does not mean that respiratory disease was mild.

The most common presentation was recurrent chest infection. It is difficult to know whether these subjects had severe or repeated episodes of respiratory sepsis leading to permanent bronchial damage that were not recalled, or whether they had an unidentified intrinsic impairment to host defence that

TABLE 5 Microbiological flora associated with each diagnosis

\begin{tabular}{|c|c|c|c|c|c|c|}
\hline Streptococcus pneumoniae & $5(15)$ & $6(19)$ & $4(20)$ & $6(32)$ & & $2(40)$ \\
\hline Pseudomonas aeruginosa & $4(12)$ & $6(19)$ & $2(10)$ & $3(16)$ & & \\
\hline Staphylococcus aureus & $3(9)$ & & $1(5)$ & & & $1(20)$ \\
\hline Candida albicans & $3(9)$ & & & & & \\
\hline Others & $\begin{array}{l}\text { Aspergillus }(n=1) \\
\text { Stenotrophomonas } \\
\text { maltophilia }(n=1)\end{array}$ & $\begin{array}{l}\text { Klebsiella } \\
\qquad(\mathrm{n}=1)\end{array}$ & $\begin{array}{l}\text { Proteus spp. } \\
\qquad(\mathrm{n}=1)\end{array}$ & & & \\
\hline $\begin{array}{l}\text { Patients with } \geqslant 2 \text { organisms } \\
\text { isolated } n\end{array}$ & 8 & 7 & 2 & 3 & 0 & 1 \\
\hline
\end{tabular}

Data are presented as n (\%), unless otherwise stated. PCD: primary ciliary dyskinesia; MRSA: methicillin-resistant Staphylococcus aureus. 
rendered them more susceptible to normal childhood infections. A proportion of patients with aspiration also presented with recurrent chest infection as their initial complaint. Symptoms of bronchiectasis can be very vague and, on many occasions, they do not point to the underlying aetiology. No aetiological cause was found in $26 \%$ of the cases. This percentage of idiopathic cases in the current series is lower compared with previously published data, although it is possible that further diagnostic testing may have uncovered alternative or missed diagnoses. The decision to terminate the investigative process was made by the consultant in charge on an individual basis, in the usual clinical manner. NiKOLAIZIK and WARNER [19] reviewed 41 cases of bronchiectasis who presented with chronic productive cough, and no cause was found in $37 \%$ of their study group. Forty-eight per cent of studied subjects from a New Zealand cohort, despite extensive investigations, had no known cause for their bronchiectasis [20]. This discrepancy may be related to the different referral pattern and study populations of the different series. Another finding from the present study is the number of cases with childhood respiratory infection as the predisposing cause of bronchiectasis. In the current cohort, only five cases were found to have developed bronchiectasis as a result of childhood pneumonia. This is in marked contrast to $\sim 70 \%$ of cases in a series from the 1960s when conditions such as measles, pertussis and TB were still prevalent [21, 22]. With the widespread use of antibiotics and early childhood vaccination, intrinsic abnormalities are becoming the more important predisposing factor for non-CF bronchiectasis. Although it is not possible to be definitive without a national survey, it seems likely that post-infective bronchiectasis is less important now than it was previously. Although referral bias is possible (given the special interests of the two centres), children were generally referred with an undiagnosed respiratory problem, rather than with a known diagnosis for tertiary management. Thus, unless by chance all the post-infective patients were retained in the district hospitals, for which there is no evidence, the current authors' conclusion that post-infective bronchiectasis is a less important category seems reasonable (although unproven). In view of this, extensive investigations for a cause of bronchiectasis are definitely warranted, because, in particular, the establishment of a diagnosis has important clinical implications. Chronic cough and recurrent wheeze were common symptoms seen in the present cohort of patients and they were frequently labelled as "difficult to control asthma". The correct diagnosis allowed the withdrawal of unnecessary and potentially dangerous medications, such as high-dose inhaled corticosteroids [23], and the implementation of airway clearance and antibiotic therapy. Rhinitis in the neonatal period and recurrent otitis media are common presenting symptoms for PCD. In a recently published case series, the mean age at diagnosis of PCD was found to be $4.4 \mathrm{yrs}$, despite a clear history of early-onset troublesome rhinitis in $76 \%$ of the cases [24]. The prompt and correct diagnosis of the condition allows early implementation of appropriate therapy, which has been shown to improve prognosis [16, 25], and the introduction of fertility counselling. Furthermore, excessive and unnecessary ENT procedures are frequently performed before the diagnosis is known, leading to considerable morbidity [18]. Managing these patients in a specialised PCD clinic leads to a dramatic reduction in the performance of these procedures, with no increased hearing loss or morbidity. In 77 patients from the current study, identifying the cause for bronchiectasis had led to a change in management, targeting treatment and intervention to the specific underlying cause. The current authors' findings are different from those reported by PASTEUR et al. [26], who found a causal factor in $47 \%$ of adults with bronchiectasis and that, in $15 \%$, identifying the cause had implications for prognosis and treatment. This discrepancy can be explained by different causes for adult and paediatric cases of non-CF bronchiectasis and a possible selection bias of the current cohort of patients.

Reported correlations between spirometry and other measurements of the severity of bronchiectasis are conflicting. In adults with bronchiectasis, WONG-YOU-CHEONG et al. [27] showed that CT scores related to FEV1. A strong correlation was also demonstrated by EDWARDS et al. [20] between FEV1 and the extent of bronchiectasis, severity of bronchial wall thickening and air trapping. However, in a study involving 27 children with non-CF bronchiectasis, FEV1 and other spirometry data were found to relate poorly to CT scores [28]. The current authors were not able to comment on the relationship between lung function and severity of bronchiectasis in the present cohort of patients, as formal CT scoring was not carried out. The effects of sputum bacteriology on lung function in adult patients have been reported. WILSON et al. [29] and HERNANDEZ et al. [30] both found that patients infected with P. aeruginosa had a greater extent of lung disease and worse lung function than uncolonised patients. In the current study, no relationships between lung function and microbial infection were demonstrated in the patients.

Whether the distribution of bronchiectasis might be sufficiently characteristic for a specific cause to be diagnosed is still controversial [7-10]. Idiopathic bronchiectasis has been reported to be predominantly basal in distribution [31]. Bronchiectasis secondary to PCD is often said to involve the middle lobe [32]. In hypogammaglobulinaemia, bronchiectasis is shown to involve mainly the lower and middle lobes and the lingula, with no cases of isolated upper lobe involvement [33]. Although certain patterns may occur more frequently in each of these examples of bronchiectasis of known aetiology, there is considerable overlap in the distribution of CT abnormalities between these diseases. In the current study, the distribution of bronchiectasis overlapped greatly among the various causes, and it was impossible to predict the specific cause simply by the distribution of the CT abnormalities. In a study by LEE et al. [8], experienced chest radiologists could accurately diagnose the cause of bronchiectasis purely on CT appearances in $45 \%$ of the cases, and the level of agreement between three radiologists had a $\kappa$-value of 0.20 . Thus, the diagnosis of the various causes of bronchiectasis cannot be reliably made on the basis of the CT appearances alone.

There are some limitations to the present study. First, the data were collected at a single time point and no longitudinal findings could be used for comparison; therefore, the current authors were not able to comment on the progression of the radiological abnormalities and disease course. In addition, repeat CT would entail extra radiology, and recent evidence suggested CT abnormalities to be poor markers of exercise capacity and functional status [34]. Secondly, the patients were a 
selected group and there was a possibility of referral bias given the special interests of the two tertiary units: immunology (Great Ormond Street Hospital for Children), and PCD (Royal Brompton Hospital). Some of the patients would not have undergone all of the investigations mentioned; for example, a patient with evidence of hypogammaglobulinaemia was unlikely to be subjected to further extensive testing. In the current series, none of the subjects had a combination of pathologies. The diagnosis of PCD was based on an examination of cilia showing abnormal beat frequency and ultrastructural abnormalities on electron microscopy. CT scanning was not part of the routine work-up, but was performed if bronchiectasis was suspected clinically. Thus, the number from the present cohort might be an underestimation of the prevalence of bronchiectasis in PCD. Likewise, this may also be true for lung damage following childhood infections, the prevalence and importance of which is hard to estimate because of likely inaccurate recall and reporting. It is possible that some of the current cases with idiopathic bronchiectasis actually had lung damage secondary to previous childhood infections. However, with the decline in incidence of these infections in the developed world, it holds true that other extrinsic insults or an underlying predisposition to respiratory infection are likely to be more important in the aetiology of bronchiectasis, and, thus, extensive investigations are warranted. Thirdly, bronchiectasis secondary to CF was not included in this study, so it cannot be determined whether CT was useful in differentiating CF bronchiectasis from bronchiectasis due to other causes. Lastly, the time period between the onset of symptoms and diagnosis was not quantified. CT could have been performed very late into the disease process where a spilling-over effect of the underlying disease may have occurred, thus resulting in the nonsignificant finding between lobar distribution of CT abnormalities and aetiology.

In summary, a large selected population of patients with bronchiectasis was reviewed, and, in $74 \%$ of cases, the aetiology could be identified. Compared with previous studies, a decreased prevalence of childhood respiratory infection was demonstrated to be a cause for the bronchiectasis. Immunodeficiency and other intrinsic abnormalities are becoming the more prevalent aetiological factors. In 77 (56\%) children, identification of a cause led to a specific change in management. Although high-resolution computed tomography allows delineation of the underlying bronchial damage, the current study suggests that it is not useful in helping to identify the underlying cause. It is proposed that these patients should be thoroughly investigated, since the identification of an underlying cause will have a significant impact on management and prognosis. Non-cystic fibrosis bronchiectasis, though rare, is still an important respiratory condition with significant morbidity. Future research should be directed at providing longitudinal clinical information and serially obtained lung function parameters to ascertain the beneficial effects of correct diagnosis and early implementation of therapy.

\section{REFERENCES}

1 Brown MA, Leman RJ. Bronchiectasis. In: Chernick V, Boat $\mathrm{T}$, eds. Kendig's disorders of the respiratory tract in children. 6th Edn. Philadelphia, W.B. Saunders, 1998; pp. 538-560.
2 Singleton R, Morris A, Redding G, et al. Bronchiectasis in Alaska Native children: causes and clinical courses. Pediatr Pulmonol 2000; 29: 182-187.

3 Eastham KM, Fall AJ, Mitchell L, Spencer DA. The need to redefine non-cystic fibrosis bronchiectasis in childhood Thorax 2004; 59: 324-327.

4 Karakoc GB, Yilmaz M, Altintas DU, Kendiril SG. Bronchiectasis: still a problem. Pediatr Pulmonol 2001; 32: 175-178.

5 Hansell DM. Bronchiectasis. Radiol Clin North Am 1998; 36: 107-128.

6 Brody AS. Cystic fibrosis: when should high-resolution computed tomography of the chest be obtained? Pediatrics 1998; 101: 1071.

7 Reiff DB, Wells AU, Carr DH, Cole PJ, Hansell DM. CT findings in bronchiectasis: limited value in distinguishing between idiopathic and specific types. AJR Am J Roentgenol 1995; 165: 261-267.

8 Lee PH, Carr DH, Rubens MB, Cole PJ, Hansell DM. Accuracy of $\mathrm{CT}$ in predicting the cause of bronchiectasis. Clin Radiol 1995; 50: 839-841.

9 Cartier Y, Kavanagh PV, Johkoh T, Mason AC, Muller NL. Bronchiectasis: accuracy of high-resolution CT in the differentiation of specific diseases. AJR Am J Roentgenol 1999; 173: 47-52.

10 Takasugi JE, Godwin JD. The airways. Semin Roentgenol 1991; 26: 175-190.

11 Rosenstein BJ, Cutting GR. The diagnosis of cystic fibrosis: a consensus statement. Cystic fibrosis foundation consensus panel. J Pediatr 1998; 132: 589-595.

12 Middleton PG, Geddes DM, Alton EW. Protocols for in vivo measurement of the ion transport defects in cystic fibrosis nasal epithelium. Eur Respir J 1994; 7: 2050-2056.

13 Phillips IJ, Rowe DJ, Dewar P, Connett GJ. Faecal elastase 1: a marker of exocrine pancreatic insufficiency in cystic fibrosis. Ann Clin Biochem 1999; 36: 739-742.

14 Rutland J, Cole PJ. Non-invasive sampling of nasal cilia for measurement of beat frequency and study of ultrastructure. Lancet 1980; 2: 564-565.

15 Narang I, Ersu R, Wilson NM, Bush A. Nitric oxide in chronic airway inflammation in children: diagnostic use and pathophysiological significance. Thorax 2002; 57: 586-589.

16 Karadag B, James AJ, Gultekin E, Wilson NM, Bush A. Nasal and lower airway level of nitric oxide in children with primary ciliary dyskinesia. Eur Respir J 1999; 13: 1402-1405.

17 Standardization of spirometry, 1994 update. American Thoracic Society. Am J Respir Crit Care Med 1995; 152: 1107-1136.

18 Hadfield PJ, Rowe-Jones JM, Bush A, Makay IS. Treatment of otitis media with effusion in children with primary ciliary dyskinesia. Eur Respir J 1997; 10: 2376-2379.

19 Nikolaizik WH, Warner JO. Aetiology of chronic suppurative lung disease. Arch Dis Child 1994; 70: 141-142.

20 Edwards EA, Metcalfe R, Milne DG, Thompson J, Byrnes CA. Retrospective review of children presenting with non cystic fibrosis bronchiectasis: HRCT features and clinical relationships. Pediatr Pulmonol 2003; 36: 87-93. 
21 Glauser E, Cook C, Harris G. Bronchiectasis: a review of 187 cases in children with follow-up pulmonary function studies in 58. Acta Paediatr Scand 1966; Suppl. 165, 1.

22 Strang C. The fate of children with bronchiectasis. Ann Intern Med 1956; 44: 630-656.

23 Drake AJ, Howells RJ, Shield JPH, Prendiville A, Ward PS, Crowne EC. Symptomatic adrenal insufficiency presenting with hypoglycaemia in children with asthma receiving high dose inhaled fluticasone propionate. BMJ 2002; 324: 1081-1083.

24 Coren ME, Meeks M, Morrison I, Buchdahl RM, Bush A. Primary ciliary dyskinesia: age at diagnosis and symptom history. Acta Paediatr 2002; 91: 667-669.

25 Ellerman A, Bisgaard H. Longitudinal study of lung function in a cohort of primary ciliary dyskinesia. Eur Respir J 1997; 10: 2376-2379.

26 Pasteur MC, Helliwell SM, Houghton SJ, et al. An investigation into causative factors in patients with bronchiectasis. Am J Respir Crit Care Med 2000; 162: 1277-1284.

27 Wong-You-Cheong JJ, Leahy BC, Taylor PM, Church SE. Airways obstruction and bronchiectasis: correlation with duration of symptoms and extent of bronchiectasis on computed tomography. Clin Radiol 1992; 45: 256-259.
28 Chang AB, Masel JP, Boyce NC, Wheaton G, Torzillo PJ. Non-CF bronchiectasis: clinical and HRCT evaluation. Pediatr Pulmonol 2003; 35: 477-483.

29 Wilson CB, Jones PW, O'Leary CJ, Hansell DM, Cole PJ, Wilson R. Effect of sputum bacteriology on the quality of life of patients with bronchiectasis. Eur Respir J 1997; 10: 1754-1760.

30 Hernandez C, Abreu J, Jimenez A, Fernandez R, Martin C. Pulmonary function and quality of life in relation to bronchial colonization in adults with bronchiectasis not caused by cystic fibrosis. Med Clin (Barc) 2002; 118: 130-134.

31 Westcott JL. Bronchiectasis. Radiol Clin North Am 1991; 29: 1031-1042.

32 Nadel HR, Stringer DA, Levison H, Turner JA, Sturgess JM. The immotile cilia syndrome: radiological manifestations. Radiology 1985; 154: 651-655.

33 Curtin JJ, Webster ADB, Farrant J, Katz D. Bronchiectasis in hypogammaglobulinaemia - a computed tomography assessment. Clin Radiol 1991; 44: 82-84.

34 Edwards EA, Narang I, Li A, Hansell DM, Rosenthal M, Bush A. HRCT lung abnormalities are not a surrogate for exercise limitation in bronchiectasis. Eur Respir J 2004; 24: 538-544. 